\title{
Utilisation de la lumière UV d'un Z-pinch pour le diagnostic d'un arc de disjoncteur industriel
}

\author{
S.S. Ciobanu, C. Fleurier, D. Hong et C. Fievet* \\ Groupe de Recherches sur l'Energétique des Milieux Ionisés, Université d'Orléans, BP. 6759, \\ 45067 Orléans cedex 2, France \\ * Centre de Recherches Merlin Gerin, 38050 Grenoble cedex, France
}

\begin{abstract}
The time evolution of concentration in copper vapor in a sulphur hexafluoride $\left(\mathrm{SF}_{6}\right)$ arc plasma in an electrical circuit breaker is obtained by an optical absorption method. The UV radiation produced by a Z-pinch discharge in Ar is absorbed by the copper atoms in their fundamental level. The optical depth is determined under the homogeneity plasma hypothesis. The analysis shows the decrease of the copper vapor concentration with time.
\end{abstract}

La présence des vapeurs métalliques produites par la vaporisation des électrodes sous l'action d'un plasma d'arc dans un disjoncteur électrique modifie les propriétés de transport (conductivité électrique et thermique) du plasma. La concentration des vapeurs peut être déterminée par une méthode d'absorption optique. $L$ 'arc électrique est produit lors de l'ouverture d'un contact entre deux électrodes réalisées en cuivre et en alliage cuivre-tungstène qui se trouvent dans une enceinte remplie de $\mathrm{SF}_{6}$ à 3 bars par le passage d'une demi-période d'un courant sinusoïdal de $50 \mathrm{~Hz}$ et d'amplitude maximum de $6 \mathrm{kA}$. La présence d'un champ magnétique produit par un aimant situé près de l'anode provoque la rotation de l'arc entre les électrodes. Cette rotation induit une forte turbulence dans la chambre d'arc, ce qui complique singulièrement les mesures optiques du fait de la perturbation de l'indice de réfraction du gaz. Il est notamment impossible d'utiliser un laser pour réaliser des mesures d'absorption car le faisceau est dévié de manière imprévisible. L'utilisation d'une source étendue à la fois spatialement et spectralement comme le Z-pinch permet de surmonter ces difficultés et d'obtenir un diagnostic raisonnable des concentrations de vapeurs. La lumière émise lors de la compression maximale du plasma de Z-pinch sous l'action du champ magnétique propre est très intense et sa distribution spectrale est proche de celle émise par un corps noir à haute température. La figure 1 montre le schéma du montage optique. L'absorption de la lumière UV du Z-pinch est notamment causée par les vapeurs de cuivre dans leur niveau fondamental d'énergie (raies d'absorption à $\lambda_{1}=324.75 \mathrm{~nm}$ et $\lambda_{2}=327.40 \mathrm{~nm}$ ). Un système à deux fibres optiques qui regardent sous des angles légèrement différents la même zone de l'arc permet de projeter sur la fente d'entrée du spectromètre d'une part la lumière émise par le plasma d'arc seul et d'autre part la lumière du Z-pinch ayant traversé le 
plasma d'arc, ce qui permet de séparer les phénomènes d'émission et d'absorption. Les spectres sont obtenus avec un OMA à $2 \mathrm{D}$. Le spectre d'absorption est:

$$
I(\lambda)=I_{0}(\lambda) \cdot e^{-t(\lambda)}
$$

où $I_{0}(\lambda)$ est le spectre d'émission du Z-pinch, $I(\lambda)$ est le spectre d'absorption et $\tau(\lambda)=\sum_{k} \tau_{k}(\lambda)$ est l'épaisseur optique totale du milieu absorbant. Pour une transition de longueur d'onde $\lambda_{0}$ l'épaisseur optique est donnée par:

$$
\tau_{\lambda_{0}}(\lambda)=\pi \pi_{0} \lambda^{2} f_{l u} P_{\lambda_{0}}(\lambda) d\left(1-\frac{g_{l} n_{u}}{g_{u} n_{l}}\right) n_{l}
$$

où $r_{0}$ est le rayon de l'électron, $f_{1 u}$ est la force d'oscillateur pour la transition entre le niveau inférieur 1 de poids statistique $g_{1}$ et densité de population $\mathrm{n}_{\mathrm{l}}$ et le niveau supérieur $\mathrm{u}$ de poids statistique $\mathrm{g}_{\mathrm{u}}$ d est l'épaisseur géométrique du plasma de CuI et $P_{\lambda_{0}}(\lambda)$ est le profile Lorentz du profil d'absorption:

$$
P_{\lambda_{0}}(\lambda)=\frac{w}{\pi} \frac{1}{w^{2}+\left(\lambda-\lambda_{0}\right)^{2}},
$$

où w est la demi-largeur du profil à la demi-hauteur. L'épaisseur optique est calculée à partir des spectres d'émission du Z-pinch et d'absorption:

$$
\tau(\lambda)=\ln \frac{I_{0}(\lambda)}{I(\lambda)} \text { où } \tau(\lambda)=\tau_{\lambda_{1}}(\lambda)+\tau_{\lambda_{2}}(\lambda) .
$$

L'approche théorique de l'épaisseur optique (Fig.3) permet de calculer le produit de l'épaisseur géométrique du plasma par la concentration des vapeurs dans le niveau fondamental (Fig.4) et, ensuite, d'estimer la concentration des vapeurs métalliques (l'épaisseur géométrique du plasma d'arc peut soit être estimée par une méthode photographique rapide, soit être calculée à partir de l'autoabsorption de certaines raies).

A

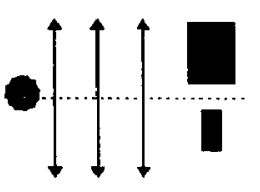

Z L

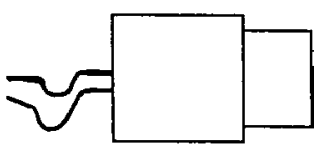

K $\quad$ F
Intensité (u.a.)

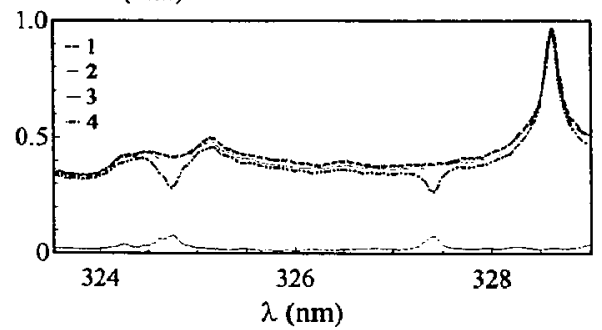

Fig.2 Spectres: 1-émission Z-pinch, 2-émission arc, 3-émission arc + absorption, 4-absorption

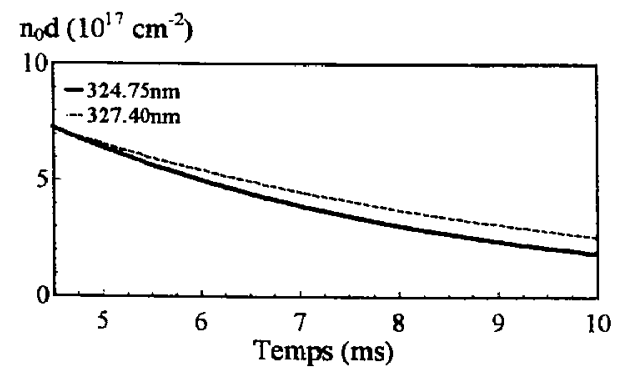

Fig.4 Evolution temporelle du produit $n_{0} d$

Tig.4 Evolution temporelle du produil nod

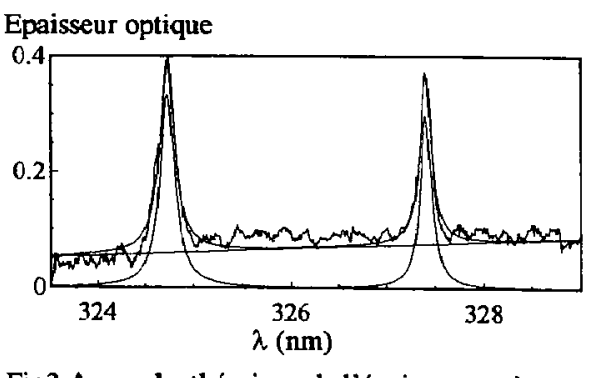

Fig3 Approche théorique de l'épaisseur optique

Fig.1 Montage expérimental: Z-décharge Zpinch, L-lentilles, K-cathode et A-anode du disjoncteur, F-fibres optiques, S-spectrometre 